Zum zweiten Mal in Folge hat die Jahrestagung der Deutschen Gesellschaft für Nuklearmedizin in der schönen Hansestadt Bremen stattgefunden. Wir blicken zurück auf eine erfolgreiche 57. Jahrestagung der DGN, an der vom 3. bis zum 6. April rund 2.100 Teilnehmer aus 16 Nationen teilgenommen haben. Der besondere Dank für diesen Erfolg und einen fachlich hochrangigen Kongress gilt dem Kongresspräsidenten, Herrn Prof. Dr. Michael Schäfers (Münster) ( $\triangleright$ Abb. 1), und dem wissenschaftlichen Komitee unter der Leitung von Herrn Prof. Dr. Markus Essler (Bonn). Diese wurden unterstützt durch das Team um den Kongresspräsidenten sowie durch das Organisationsteam der vokativ $\mathrm{GmbH}$, in dessen Händen die Kongressorganisation lag.

Den Auftakt der Jahrestagung bildete traditionell die Wolfgang Becker-Gedächtnis-
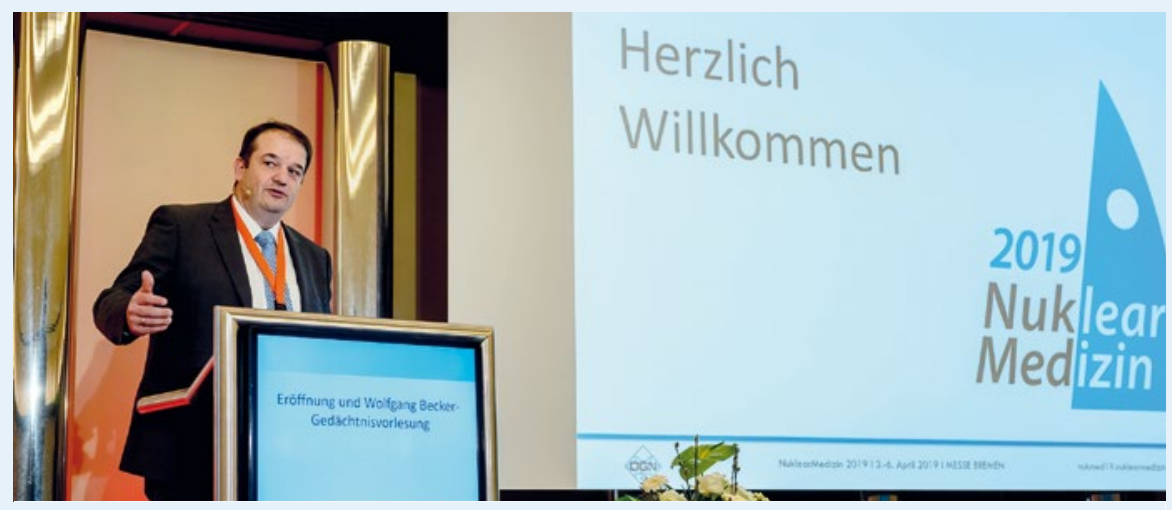

- Abb. 1 Kongresspräsident Prof. Dr. Michael Schäfers (Münster) (Quelle: Frank Pusch, Bremen)

vorlesung. Hierfür konnte in diesem Jahr unser korrespondierendes Mitglied Herr Prof. Dr. Kurt Werner Schmid (Essen) gewonnen werden, der zum Thema „Pathologie und Nuklearmedizin - Stärken und Perspektiven für eine enge Zusammenarbeit?“ sprach ( $>$ Abb. 2). Insgesamt wurden auf der NuklearMedizin 2019 in Bremen 126 Vorträge und 147 Poster präsentiert. Besonders hervorzuheben sind die sieben LeuchtturmSitzungen, die sich wie ein roter Faden durch den Kongress zogen und in denen richtungs- 


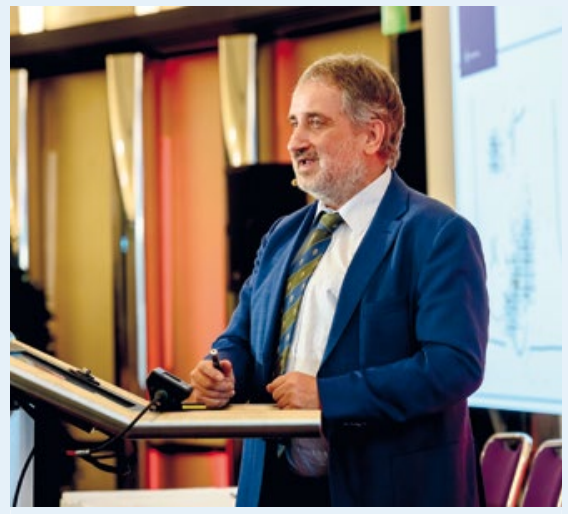

Abb. 2 Prof. Dr. Kurt Werner Schmid (Essen), Referent der Wolfgang BeckerGedächtnisvorlesung (Quelle: Frank Pusch, Bremen)

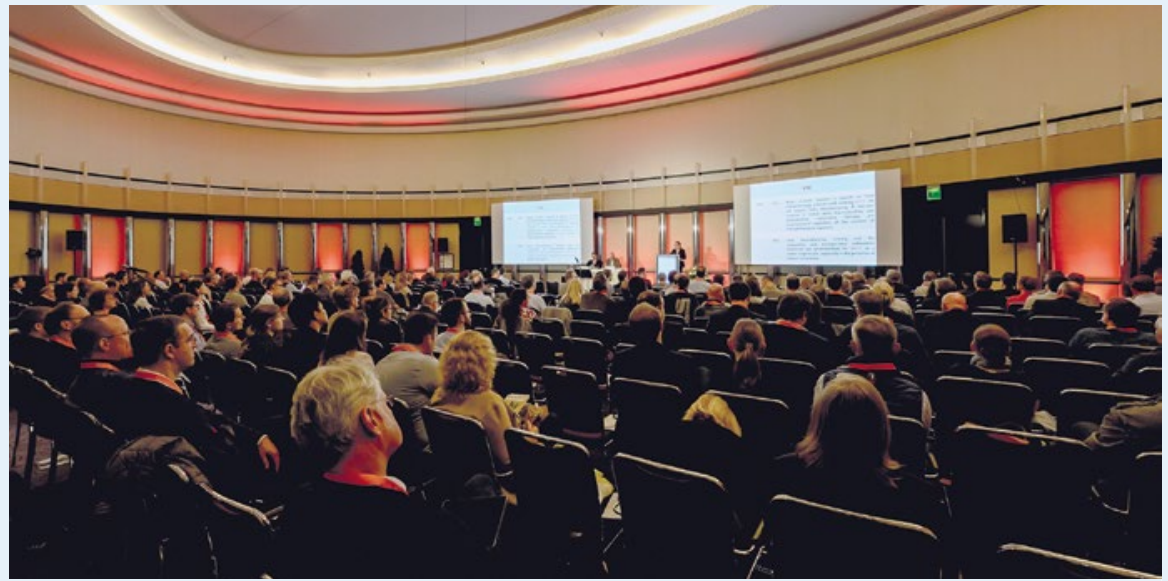

- Abb. 3 Der Erfolg der NuklearMedizin 2019 zeigte sich auch in den gut besuchten Veranstaltungen (Quelle: Frank Pusch, Bremen).

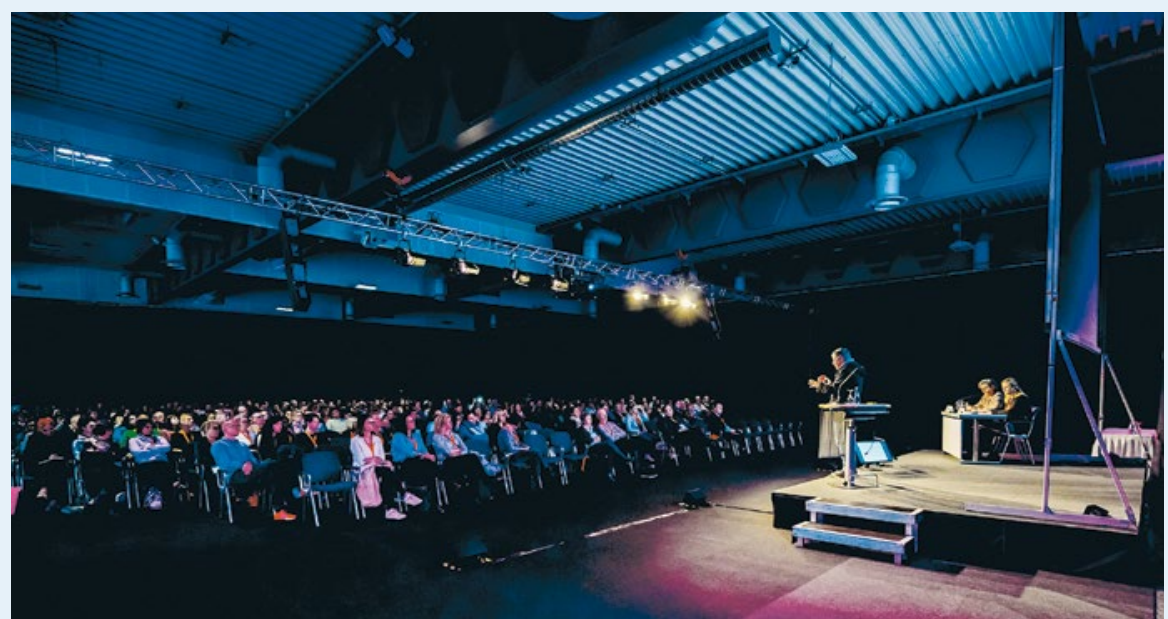

Abb. 4 Das MTRA-Programm verzeichnete auch in diesem Jahr wieder erfreulich hohe Besucherzahlen (Quelle: Frank Pusch, Bremen).

Auswertung hinterfragt und eingeordnet werden.

Aufgrund der großen Resonanz in den Vorjahren haben auf der Jahrestagung sowohl wieder ein DRG-Workshop als auch zwei Nuklearmedizin- und Radiologie-Foren stattgefunden. Beim DRG-Workshop „Stationäre Radionuklidtherapien - der Tag der Abrechnung“ hat Dr. Wolfgang Fiori ausgewählte Ergebnisse der DRG-Fallkostenkalkulation für 2019 vorgestellt und Auswirkungen der unterschiedlichen Finanzierungsmethoden erläutert.

Die Nuklearmedizin- und Radiologie-Foren wurden gemeinsam von der Deutschen Gesellschaft für Nuklearmedizin (DGN) und der Deutschen Röntgengesellschaft (DRG) unter Federführung der Interdisziplinären Arbeits- gemeinschaft für hybride Bildgebung (ID AG Hybride BG) organisiert. Unter der Leitung von Prof. Dr. Lale Umutlu (Essen) und Prof. Dr. Ken Herrmann (Essen) gab die Veranstaltung einen Überblick zu bildgebenden Untersuchungsverfahren mit PET/CT und PET/MRT. Renommierte Vertreter der Fächer haben hier zu ihren jeweiligen Schwerpunkten referiert.

Das MTRA-Programm wies auch in diesem Jahr attraktive Angebote auf und verzeichnete ebenfalls wieder erfreulich hohe Besucherzahlen. Es bestand aus 13 freien Vortragspräsentationen sowie sechs Fortbildungsveranstaltungen ( $\mathbf{A} \mathbf{b b} \mathbf{4} \mathbf{4})$. Zudem hatten die MTRA Gelegenheit, sich bei der MTRA-Lehrveranstaltung „Nuklearmedizin Therapien auf Station - von der Voruntersuchung bis zur Entlassung des Patienten“ 


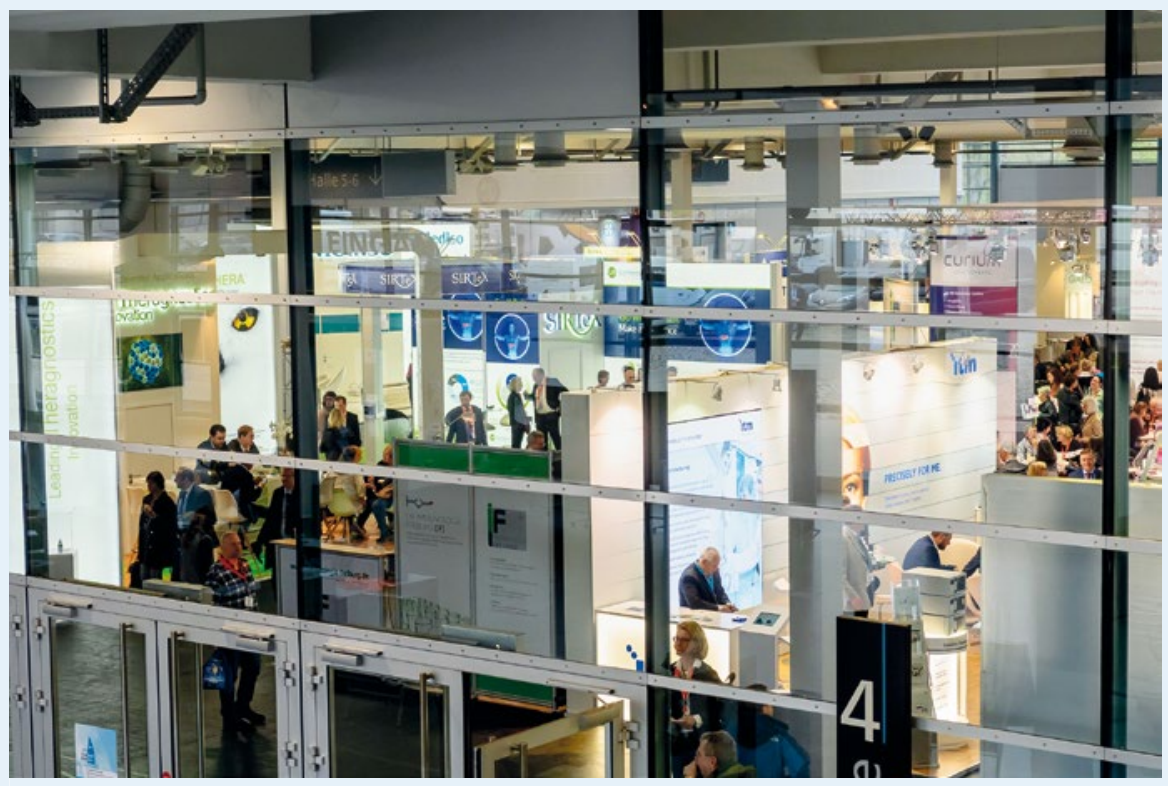

Abb. 5 Die Industrieausstellung bot den Besuchern und Ausstellen Gelegenheit zum fachlichen Austausch (Quelle: Frank Pusch, Bremen).

zu informieren. Die organisatorische Leitung der MTRA-Veranstaltungen lag erneut beim Vorstand der AG-MTM der DGN.

Mit 57 Ausstellern aus elf Ländern konnte auch die Fachausstellung der Industrie erneut eine hohe Beteiligung verbuchen ( Abb.5). Zahlreiche Teilnehmer besuchten die Messestände und nutzten die Gelegenheit zum regen Austausch mit den Industrieunternehmen und Fachverbänden. Fünf Lunchsymposien mit interessanten Programmen rundeten darüber hinaus das Tagungsgeschehen ab.

Wir bedanken uns bei allen Teilnehmern und Mitwirkenden für ihren Beitrag zu einem gelungenen und erfolgreichen Kongress. Mit dem Fotorückblick können Sie die diesjährige Tagung Revue passieren lassen. Sie finden ihn auf der Kongresshomepage der NuklearMedizin 2020 (nukmed20.nuklearmedizin.de).

\section{Zukunftsworkshop Nuklearmedizin}

Wie sieht die Nuklearmedizin der Zukunft aus? Spannende Perspektiven in diesem Fachgebiet wurden beim Zukunftsworkshop am 6. April 2019 zum Abschluss der NuklearMedizin 2019 diskutiert - moderiert von der Journalistin Natascha Plankermann.
Organisiert wurde diese erfolgreiche Veranstaltung von Prof. Dr. Dr. Lutz Stefan Freudenberg (Grevenbroich) sowie Prof. Dr. Ken Herrmann (Essen) ( $\triangleright$ Abb. 6).

Über die vier brennendsten Themen, die bei dem Zukunftsworkshop im Mittelpunkt standen, hatten die Mitglieder der DGN bereits im Vorfeld der Veranstaltung abgestimmt: Zukunftsprofil Nuklearmedizin, Interdisziplinarität, die Bedeutung von Künstlicher Intelligenz und Big Data für die Nuklearmedizin sowie die Möglichkeiten, den Nachwuchs für die Nuklearmedizin zu begeistern.

Vor Ort in Bremen haben die rund 300 Teilnehmer sich dann lebhaft an der Suche nach Lösungen und zukünftigen Zielen im Hinblick auf diese vier Themen beteiligt und sich mehrheitlich dafür ausgesprochen, das Format des Zukunftsworkshops auch für zukünftige Jahrestagungen aufzugreifen.

\section{Vorkongress-Symposium zum Thema Schilddrüsenkarzinom}

Mit knapp 300 Besuchern ist das Vorkongress-Symposium der Jahrestagung auf sehr großes Interesse bei den Kongressteilnehmern gestoßen ( $\triangleright$ Abb. 7 ).
Zum Auftakt der NuklearMedizin 2019 widmete sich das Symposium am 3. April 2019 dem Thema „Therapie des metastasierten Schilddrüsenkarzinoms: Aktuelle Leitlinien - aktuelle Kontroversen“. Die gemeinsame wissenschaftliche Leitung lag in den Händen von Prof. Dr. Markus Essler (Bonn), Prof. Dr. mult. Andreas Bockisch (Essen), Prof. Dr. Markus Luster (Marburg), Prof. Dr. Klemens Scheidhauer (München) sowie Prof. Dr. Matthias Schmidt (Köln).

Neben erfahrenen Nuklearmedizinern konnten für das Vorkongress-Symposium auch Referenten aus den anderen Fächern gewonnen werden, die an der Behandlung des Schilddüsenkarzinoms beteiligt sind (Endokrinologie, Onkologie, Chirurgie, Pathologie). So ist es in Bremen gelungen, dieses wichtige Thema interdisziplinär und auch kontrovers zu diskutieren.

\section{Verleihung der korres- pondierenden Mitgliedschaft der DGN}

Herrn Prof. Dr. Kurt Werner Schmid (Essen) wurde im Rahmen der Eröffnungsfeier der NuklearMedizin 2019 in Bremen die korrespondierende Mitgliedschaft der DGN verliehen ( $\triangleright$ Abb. 8). Unsere Fachgesellschaft würdigt damit seine Verdienste für das Fachgebiet Nuklearmedizin sowie sein Engagement auf dem Gebiet der histopathologischen und molekularen Diagnostik von Krankheits- und Therapiemechanismen.

\section{Preis der Dr. Dagobert Nitz Stiftung}

Bereits zum vierten Mal konnte in diesem Jahr der Preis der Dr. Dagobert Nitz Stiftung vergeben werden. Herr Prof. Dr. Wolfgang Weber (München), als Mitglied des Preiskomitees, überreichte den Preis im Rahmen der Schlussveranstaltung an Herrn Prof. Dr. Dr. Frederik Verburg (Marburg) für seine Forschung auf dem Gebiet der Schilddrüsenerkrankungen ( Abb. 9).

Herrn Professor Verburg gelten die Glückwünsche unserer Fachgesellschaft zu dieser Auszeichnung. 

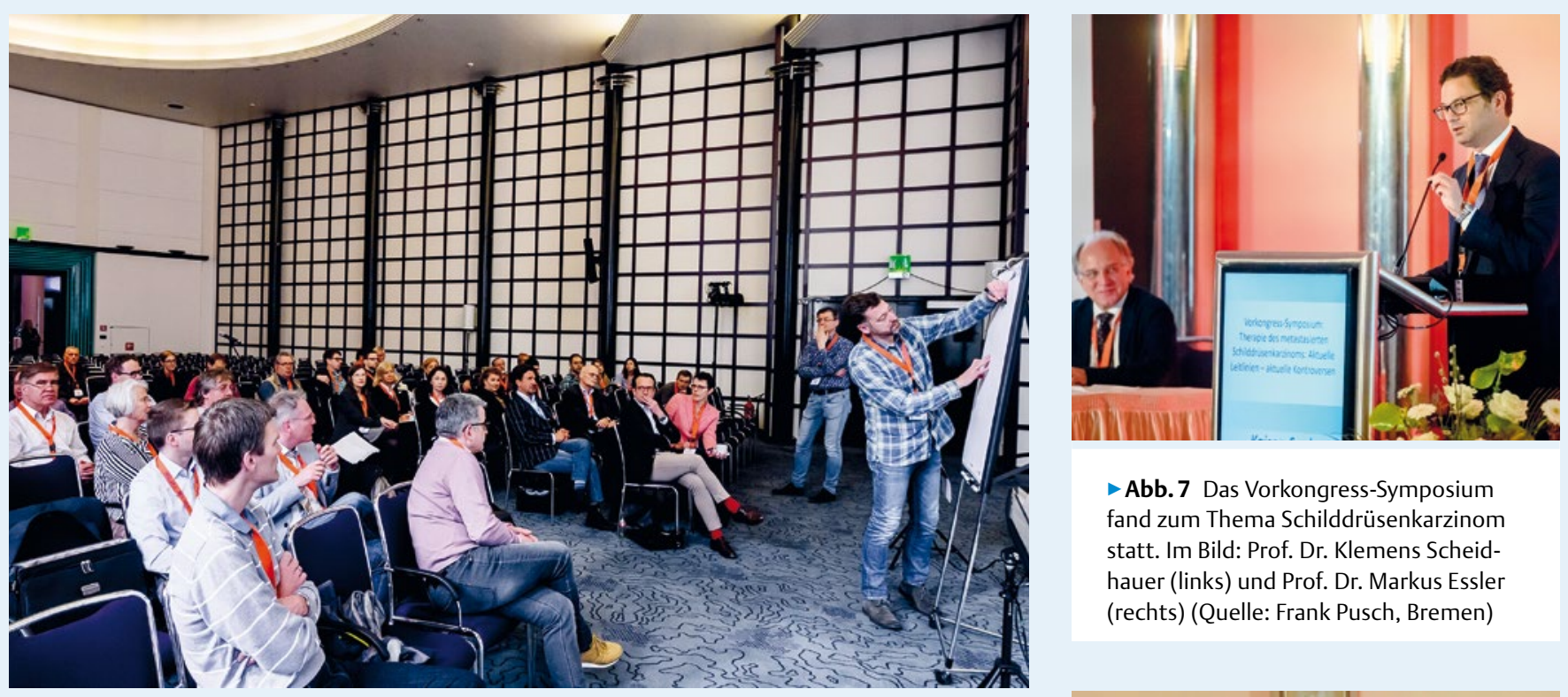

Abb. 7 Das Vorkongress-Symposium fand zum Thema Schilddrüsenkarzinom statt. Im Bild: Prof. Dr. Klemens Scheidhauer (links) und Prof. Dr. Markus Essler (rechts) (Quelle: Frank Pusch, Bremen)
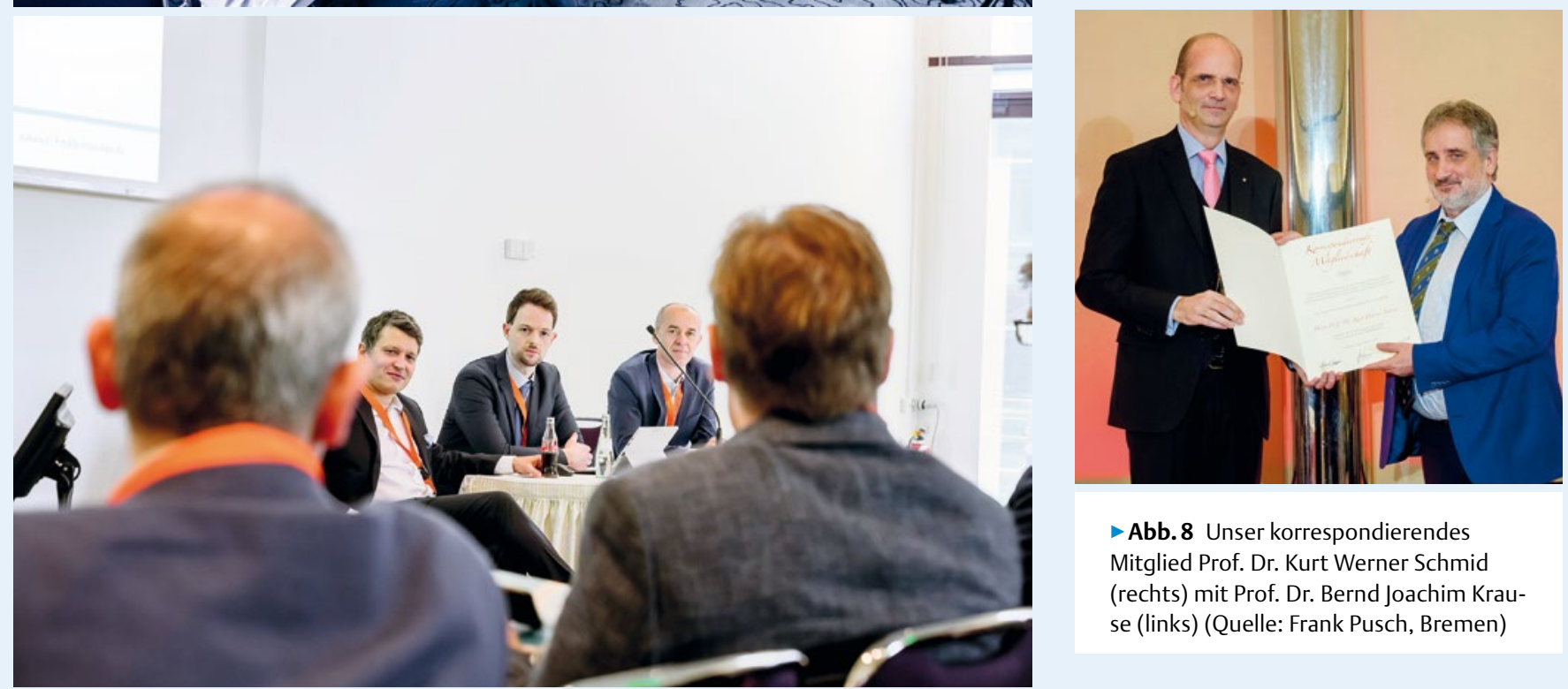

Abb. 8 Unser korrespondierendes Mitglied Prof. Dr. Kurt Werner Schmid (rechts) mit Prof. Dr. Bernd Joachim Krause (links) (Quelle: Frank Pusch, Bremen)
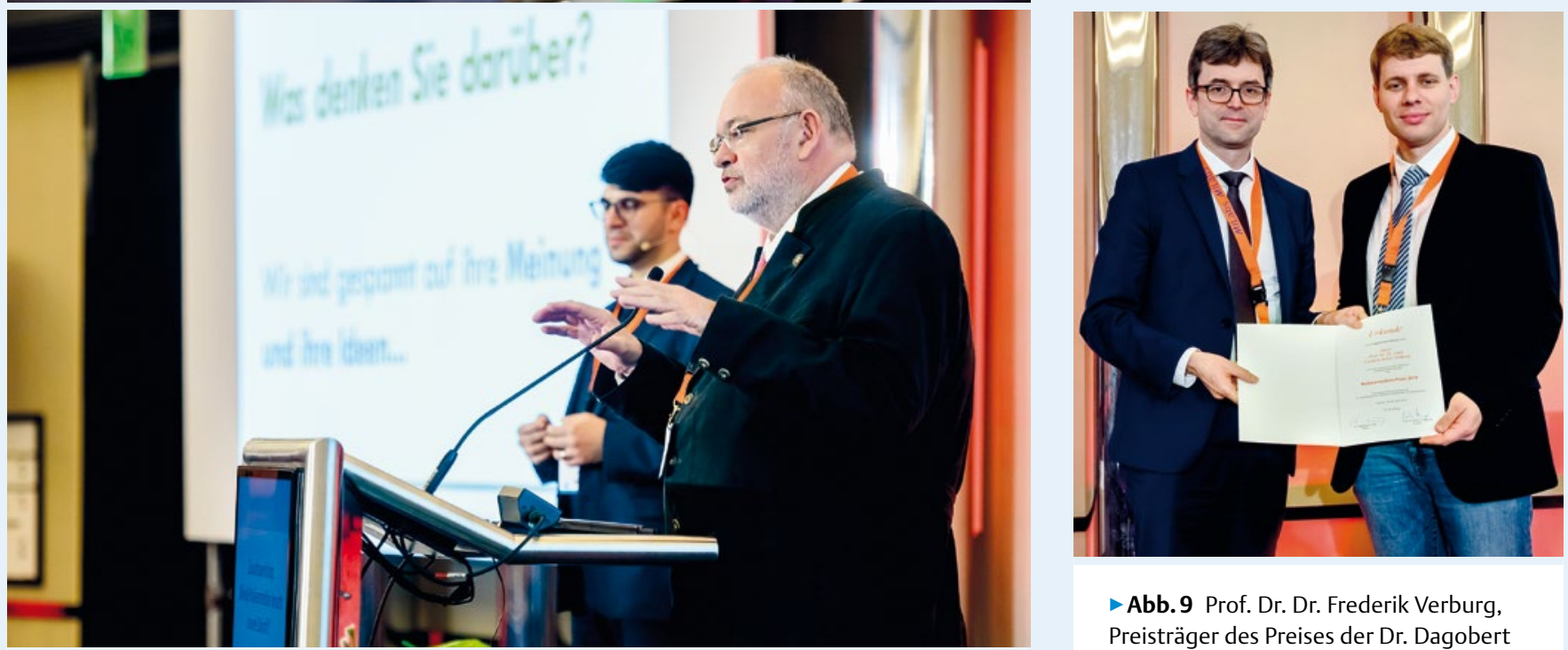

- Abb.9 Prof. Dr. Dr. Frederik Verburg, Preisträger des Preises der Dr. Dagobert Nitz Stiftung, mit Prof. Dr. Wolfgang Weber (von rechts nach links) (Quelle: Frank Pusch, Bremen)

Abb. 6 Spannende Perspektiven unseres Fachgebiets wurden beim Zukunftsworkshop diskutiert (Quelle: Frank Pusch, Bremen). 
Die Deutsche Gesellschaft für Nuklearmedizin dankt dem Stifter, Herrn Dr. Dagobert Nitz (München), für die unserem Fachgebiet entgegengebrachte Wertschätzung und freut sich sehr über die Förderung durch die Dr. Dagobert Nitz-Stiftung.

\section{Preisverleihungen im Rahmen der NuklearMedizin 2019}

Auch in diesem Jahr wurden während der Jahrestagung zudem zahlreiche Fach- und Förderpreise vergeben:

Den Georg von Hevesy-Preis der DGN erhielt Herr James Thackeray (Hannover) für seine Arbeit „Myocardial Inflammation Predicts Remodeling and Neuroinflammation After Myocardial Infarction“.

Der Förderpreis der DGN für Leistungen auf dem Gebiet der nuklearmedizinischen Therapie ging an Herrn Dr. Peter Kletting (Ulm) für seine Arbeit „Modeling and Predicting Tumor Response in Radioligand Therapy“.

Den Sonderpreis der DGN zur Förderung der Digitalen Transformation des Fachgebietes Nuklearmedizin erhielt Frau Ganna Blazhenets (Freiburg) für ihre Arbeit „Principal component analysis of brain metabolism predicts development of Alzheimer's dementia”.

Den Cuno Winkler-Preis der DGN erhielt Herr Conrad-Amadeus Voltin (Köln) für seine Arbeit „Value of bone marrow biopsy in Hodgkin lymphoma patients staged by FDG PET: results from the German Hodgkin Study Group trials HD16, HD17, and HD18“.

Die Auszeichnung „Junge Talente“ ging an Herrn Dr. Johannes Schwenck (Tübingen) für seinen Vortrag „In Vivo Imaging of Tumor Senescence with a novel beta-Galactosidase specific PET Tracer“ (L5).

Mit dem Preis für das beste wissenschaftliche Poster der Tagung wurde Herr Dr. Armin Fril-

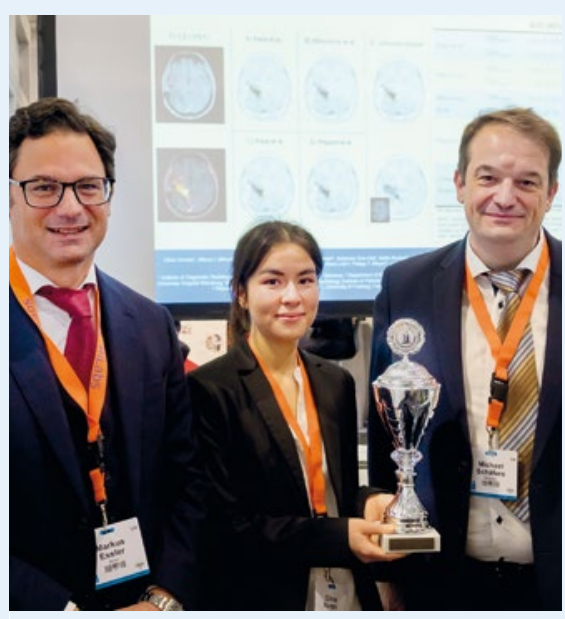

- Abb. 10 Erstmals wurde im Rahmen der diesjährigen Tagung der LeuchtfeuerPosterpreis der DGN verliehen. Er ging an Frau Dr. Olivia Kertels (Würzburg) (Quelle: Frank Pusch, Bremen)

le (Leipzig) für seinen Beitrag „Brown adipose tissue activation quantified by positron emission tomography/computed tomography describes pulmonary cachexia in COPD patients“ (P124) ausgezeichnet.

Erstmals wurde im Rahmen der diesjährigen Tagung der Leuchtfeuer-Posterpreis der $D G N$ verliehen. Dieser ging an Frau Dr. Olivia Kertels (Würzburg) für ihr Poster „Clinical utility of different approaches for detection of late pseudoprogression in glioblastoma with O-(2-[18 F]fluoroethyl)-L-tyrosine PET" (P86) (॰ Abb. 10).

Der Preis für den besten Vortrag der MTRA-Tagung wurde an Frau Cornelia Pötzsch (Leipzig) für ihren Vortrag „Prävention von aktiviertem braunen Fett in der F18-FDG-PET (PET)-Bildgebung bei Kindern und Jugendlichen - Effektivste Maßnahmen?" (TV8) vergeben.

Zudem hat der Thieme-Verlag den Nuklearmedizin-Preis 2019 an Herrn Prof. Dr. Markus Dietlein (Köln) verliehen. Er erhielt diese Auszeichnung für seine besonderen Verdienste für die Zeitschrift Nuklearmedizin - NuclearMedicine ( $\triangleright$ Abb. 11).

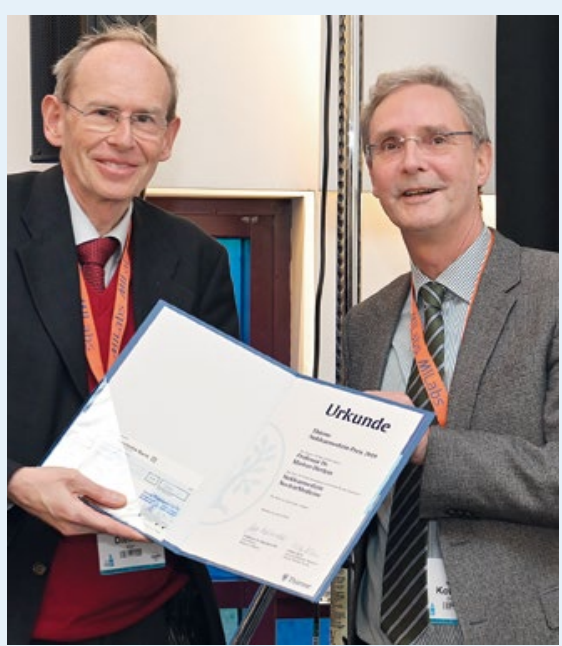

-Abb. 11 Prof. Dr. Markus Dietlein (Köln) erhält den Nuklearmedizin-Preis 2019 von Prof. Dr. Jörg Kotzerke (Dresden) (Quelle: Frank Pusch, Bremen).

Die DGN gratuliert allen Preisträgern herzlich!

\section{NuklearMedizin 2020}

In der Schlussveranstaltung lud Herr Prof. Dr. Markus Essler (Bonn) als Kongresspräsident zur 58. Jahrestagung der DGN im kommenden Jahr nach Leipzig ein.

Die DGN freut sich auf ein Wiedersehen vom 22. bis zum 25. April 2020 zur NuklearMedizin 2020 in Leipzig!

VERANTWORTLICH FÜR DEN INHALT

\section{Deutsche Gesellschaft für -}

Nuklearmedizin e. V. (DGN)

Prof. Dr. Bernd Joachim Krause

(Präsident)

Nikolaistr. 29, 37073 Göttingen

E-Mail: office@nuklearmedizin.de

\section{Schriftführer:}

Prof. Dr. Peter Bartenstein

E-Mail: office@nuklearmedizin.de 